\title{
Hardening Effects of the TeV-scale Cosmic Ray Proton and Helium Spectra
}

\author{
Jihyeun Bang* \\ Author affiliation: Department of Physics, Seoul National University, 1 Kwan-Ak Ro, Kwan-Ak \\ Gu, Seoul, Republic of Korea \\ E-mail: coolbang115esnu.ac.kr \\ Yong-Yeon Keum \\ Center for Theoretical Physics, Department of Physics, Seoul National University, 1 Kwan-Ak \\ Ro, Kwan-Ak Gu, Seoul, Republic of Korea \\ E-mail: yykeum2011@snu.ac.kr
}

\begin{abstract}
We suggest a new way to understand cosmic ray proton and helium spectrum around $\mathrm{TeV}$-scale energy by introducing discrete local sources. The source of diffusion equation in cosmic ray propagation is set to be a point source given by delta function, contrary to conventional models which have continuous sources. We calculate the spectrum of proton and helium originated in close and young supernovas. By combining the solutions from discrete source and faraway continuous sources, we found cosmic ray propagation parameters of both helium and proton which explain the discrepancy between conventional model of cosmic ray and recent measurements of CREAM, PAMELA, and AMS-02 experiments, without any additional assumptions.
\end{abstract}

The 39th International Conference on High Energy Physics (ICHEP2018)

4-11 July, 2018

Seoul, Korea

${ }^{*}$ Speaker. 

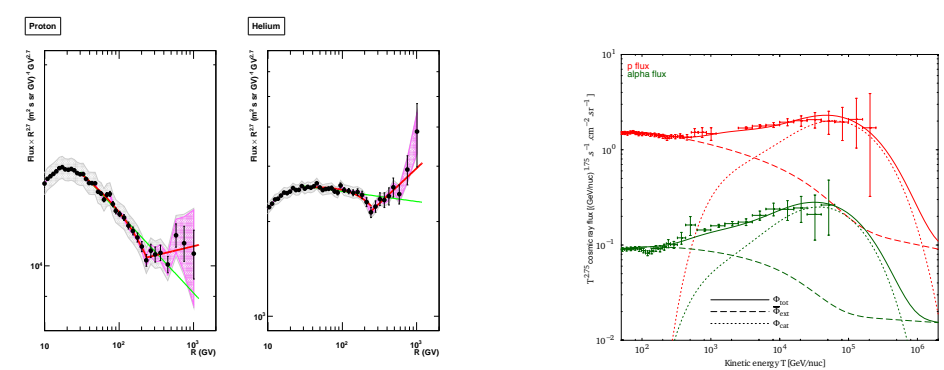

Figure 1: (A) Proton and Helium spectrum reported by PAMELA Collaboration [2], (B) Our result: Proton (upper curve) and helium (lower curve) spectra in the range extending from $50 \mathrm{GeV} /$ nuc to $100 \mathrm{TeV} / \mathrm{nuc}$, for the propagation parameters of model A in ref.[4], giving the best fit to the CREAM [1] and PAMELA [2] data : supernovae explosion rate $v=0.8$ century $^{-1}$. Solid lines show the total flux, short-dashed lines show the flux due to the sources of the catalog, and the long-dashed curve the flux due to the rest of the sources.

Discrepant hardenings in the $\mathrm{TeV}$ scale cosmic ray proton-helium nuclei spectra was reported by CREAM [1] and PAMELA [2] experiments. Recently this anomalous phenomena was also confirmed by AMS02 collaboration [3]. Observations have pointed towards an excess in the CR proton and helium fluxes above $250 \mathrm{GeV} /$ nuc. The single power-law hypothesis is rejected at $95 \%$ C.L. In this poster, we explain that the proton and helium spectral hardening above $250 \mathrm{GeV} / \mathrm{nuc}$ can be attributed to local sources of cosmic rays, whose presence is associated to known supernova remnants (SNR) and pulsars. In the conventional model of CR propagation, these are treated as a jelly spreading over the Galactic disk and continuously accelerating cosmic rays. However in our model, we used the Green Function Method for the point like sources. As shown in Figure 1(B), this excellent agreement makes us confident that the proton and helium anomaly can actually be explained by existing point-like local sources which have been extracted from SNR and pulsar surveys. To understand more details of our model, we suggest you to touch with our results [4].

\section{Acknowledgments}

This research work is supporting by the Basic Science Research Program through the National Research Foundation of Korea (NRF-2017R1D1A1B03035250).

\section{References}

[1] H.S. Ahn et al. (CREAM collaboration), The Astrophysical Journal 714 L89-L93 (2010); The Astrophysical Journal 728122 (2011).

[2] O. Adriani et al. (PAMELA collaboration), Science 33269 (2011).

[3] M. Aguilar et al. (AMS02 collaboration), Phys. Rev. Lett. vol. 114, p. 171103 (2015); Phys. Rev. Lett. vol. 115, no. 21, p. 211101 (2015).

[4] G. Bernard, T. Delahaye, Y.-Y. Keum, W. Liu, P. Salati, and R. Taillet, Astronomy \& Astrophysics 555, A48 (2013); Yong-Yeon Keum and P. Salati, PARAMANA-journal of physics, Vol 86, No 2, pp369-377 (2016); Jihyeun Bang and Yong-Yeon Keum, PoS Proceedings of ICHEP2018, 544(2018). 